\title{
Surveillance de la maladie de Lyme au Canada, de 2009 à 2015
}

\author{
S Gasmi', NH Ogden², LR Lindsay ${ }^{3}$, S Burns ${ }^{4}$, S Fleming 5 , J Badcock6, S Hanan ${ }^{6}$, C Gaulin7, \\ MA Leblanc ${ }^{7}$, C Russell ${ }^{8}$, M Nelder ${ }^{8}$, L Hobbs ${ }^{8}$, S Graham-Derham ${ }^{9}$, L Lachance ${ }^{10}$, AN Scott ${ }^{11,12}$, \\ E Galanis ${ }^{13}$, JK Koffi ${ }^{1 *}$
}

\section{Résumé}

Objectif : Résumer sept années de données de surveillance sur les cas de maladie de Lyme signalés au Canada de 2009 à 2015.

Méthodologie : Nous décrivons l'incidence des cas signalés de la maladie de Lyme au fil du temps, selon la répartition saisonnière et géographique, et en fonction des caractéristiques démographiques et cliniques. Les écarts entre les groupes d'âge, les sexes et les années ont été explorés au moyen du modèle de régression logistique afin de mieux comprendre les facteurs de risque démographique potentiels associés à la présence de la maladie de Lyme.

Résultats : Le nombre de cas de la maladie de Lyme signalés a plus que sextuplé, passant de 144 en 2009 à 917 en 2015, principalement en raison de la hausse des infections contractées au Canada. La plupart des infections contractées localement ont été signalées entre mai et novembre. Une hausse de l'incidence de la maladie de Lyme a été observée au Manitoba et dans les provinces situées plus à l'Est, ce qui correspond aux connaissances que nous possédons sur l'expansion de l'aire de répartition des tiques vectrices dans cette région. Dans les provinces de l'Ouest, l'incidence est restée faible et stable. Tous les cas signalés par I'Alberta, la Saskatchewan et Terre-Neuve-et-Labrador ont été contractés à l'extérieur des provinces en question, au Canada ou à l'étranger. La maladie de Lyme est associée à une distribution bimodale liée à l'âge, avec un pic chez l'enfant âgé de 5 à 9 ans et un autre chez l'adulte âgé de 45 à 74 ans. Les symptômes les plus couramment observés sont l'érythème migrant (74,2 \%) et l'arthrite (35,7\%). Des différences de fréquence des manifestations cliniques ont été observées entre les groupes d'âge et les années étudiées.

Conclusion : L'incidence de la maladie de Lyme continue d'augmenter au Canada, tout comme la portée géographique des tiques qui transportent la bactérie responsable de la maladie. La surveillance continue, les stratégies de prévention ainsi que la détection et le traitement précoces de la maladie permettront de réduire au minimum les répercussions de la maladie de Lyme au Canada.

Citation proposée : Gasmi S, Ogden NH, Lindsay LR, Burns S, Fleming S, Badcock J, Hanan S, Gaulin C, Leblanc MA, Russell C, Nelder M, Hobbs L, Graham-Derham S, Lachance L, Scott AN, Galanis E, Koffi JK. Surveillance de la maladie de Lyme au Canada, de 2009 à 2015. Relevé des maladies transmissibles au Canada. 2017;43(10):219-25. https://doi.org/10.14745/ccdr.v43i10a01f

\section{Affiliations}

Centre des maladies infectieuses d'origine alimentaire environnementale et zoonotique, Agence de la santé publique du Canada, Saint-Hyacinthe (Québec)

2 Division Science des risques pour la santé publique, Laboratoire national de microbiologie, Agence de la santé publique du Canada, Saint-Hyacinthe (Québec)

${ }^{3}$ Laboratoire national de microbiologie, Agence de la santé publique du Canada, Winnipeg (Manitoba)

${ }^{4}$ Programme de lutte contre les maladies transmissibles, ministère de la Santé et du Mieux-être, Charlottetown (Île-du-Prince-Édouard)

${ }^{5}$ Direction générale de la santé publique, ministère de la Santé et du Mieux-être de la Nouvelle-Écosse, Halifax (Nouvelle-Écosse)

${ }^{6}$ Bureau du médecin-hygiéniste en chef, ministère de la Santé du Nouveau-Brunswick, Fredericton (Nouveau-Brunswick)

${ }^{7}$ Direction de la protection, ministère de la Santé et des Services sociaux Québec (Québec)

${ }^{8}$ Maladies entériques, zoonotiques et à transmission vectorielle, Santé publique Ontario, Toronto (Ontario)

9 Direction générale de la lutte contre les maladies transmissibles, Santé, Aînés et Vie active du Manitoba, Winnipeg (Manitoba)

${ }^{10}$ Direction générale de la surveillance et de l'évaluation, ministère de la Santé de I'Alberta, Calgary (Alberta)

${ }^{11}$ Direction générale de la promotion de la santé et du mieux-être, ministère de la Santé de l'Alberta, Edmonton (Alberta)

${ }^{12}$ Direction générale des analyses et des rapports sur le rendement ministère de la Santé de l'Alberta, Edmonton (Alberta)

${ }^{13}$ Maladies entériques, zoonotiques et à transmission vectorielle, Centre de contrôle des maladies de la Colombie-Britannique, Vancouver (Colombie-Britannique)

\section{Introduction}

La maladie de Lyme, causée par le spirochète Borrelia burgdorferi sensu stricto, est la maladie à transmission vectorielle la plus souvent signalée en Amérique du Nord. La maladie de Lyme est transmise par les tiques à pattes
*Correspondance : juleskonan.koffi@ canada.ca noires Ixodes scapularis dans l'est et le centre du Canada, et Ixodes pacificus dans l'ouest du Canada (1). La maladie de Lyme est une infection multisystémique qui évolue en plusieurs stades (2). Au stade précoce, un érythème cutané migrant apparaît dans les 30 jours suivant l'infection, à l'endroit de la piqûre par une tique infectée, chez environ $70 \%$ des personnes touchées 
(3). L'éruption cutanée peut être accompagnée de symptômes pseudo-grippaux comme de la fièvre, de la fatigue, des maux de tête et des douleurs musculaires ou articulaires. Si l'infection n'est pas traitée, B. burgdorferi peut se disperser dans la circulation sanguine dans les trois mois suivant l'infection (2). Les manifestations du stade disséminé précoce de la maladie de Lyme comprennent des lésions d'érythème migrant multiples secondaires, des symptômes neurologiques (p. ex. paralysie du nerf facial et méningite) et des symptômes cardiaques (p. ex. bloc cardiaque) lesquels peuvent, dans de rares cas, être mortels (4). Au fil des mois, voire des années, la maladie de Lyme au stade disséminé précoce non traitée peut évoluer vers le stade disséminé tardif, dont l'arthrite est la manifestation la plus courante (3).

En 2004, environ 40 cas humains de la maladie de Lyme ont été signalés au Canada (1). En 2009, la maladie de Lyme est devenue une maladie à déclaration obligatoire à l'échelle nationale, les ministères de la santé provinciaux et territoriaux devant déclarer les cas diagnostiqués sur le plan clinique au Système de surveillance des maladies à déclaration obligatoire du Canada (SSMDOC) de l'Agence de la santé publique du Canada (5). En 2010, le Système de surveillance accrue de la maladie de Lyme (le Système), conçu par un groupe de travail du Réseau pancanadien de santé publique, a été mis en œuvre. Ce système vise à mieux repérer les populations canadiennes à risque en analysant des renseignements additionnels en plus de ceux habituellement transmis au SSMDOC (6). Cette étude s'aligne sur les trois piliers du cadre fédéral relatif à la maladie de Lyme, dont I'un d'entre eux vise " l'établissement d'un programme national de surveillance médicale qui utilise les données recueillies par l'Agence pour suivre adéquatement les taux d'incidence et les coûts économiques liés à la maladie de Lyme » (7).

L'objectif de cette étude est de résumer les sept années de données de surveillance sur les cas de la maladie de Lyme déclarés au Canada de 2009 à 2015, afin de déterminer l'incidence de la maladie au fil du temps, selon la répartition saisonnière et géographique, et en fonction des caractéristiques démographiques et cliniques.

\section{Méthodologie}

\section{Définition de cas}

La définition des cas de la maladie de Lyme à l'échelle nationale en 2009 utilisée dans le cadre de l'étude (8) est présentée dans le tableau 1.

\section{Sources des données}

Les renseignements sur les cas déclarés de la maladie de Lyme de 2009 à 2015 ont été obtenus auprès du SSMDOC et du Système. Le SSMDOC ne collecte que des données démographiques (âge et sexe), la date de l'épisode et la classification des cas. Le Système recueille des données supplémentaires, notamment les suivantes:

- emplacement géographique possible de l'infection, y compris les infections contractées localement et les cas liés à un voyage;

- manifestations cliniques;

- résultats des tests de laboratoire.
Tableau 1 : Définition des cas de la maladie de Lyme à l'échelle nationale en 2009

\begin{tabular}{|c|c|}
\hline Cas confirmé & Cas probable \\
\hline $\begin{array}{l}\text { Manifestation clinique de la } \\
\text { maladie et confirmation en } \\
\text { laboratoire par : } \\
\text { - I'isolement de Borrelia } \\
\text { burgdorferi dans un échantillon } \\
\text { clinique approprié } \\
\text { OU } \\
\text { - Détection d'ADN de } \\
\text { B. burgdorferi par réaction en } \\
\text { chaîne de la polymérase (PCR) } \\
\text { OU } \\
\text { - Manifestations cliniques de } \\
\text { la maladie avec antécédents } \\
\text { de résidence ou de voyage } \\
\text { dans une région endémique } \\
\text { et résultats de laboratoire } \\
\text { indiquant une infection, c.-à-d. } \\
\text { test sérologique positif selon } \\
\text { les critères de l'approche en } \\
\text { deux étapes (épreuve ELISA et } \\
\text { méthode de transfert Western } \\
\text { blot) }\end{array}$ & $\begin{array}{l}\text { Manifestations cliniques de la } \\
\text { maladie sans antécédents de } \\
\text { résidence ou de voyage dans } \\
\text { une région endémique, mais } \\
\text { avec des résultats de laboratoire } \\
\text { indiquant une infection au } \\
\text { moyen des méthodes suivantes : } \\
\text { - Test sérologique positif selon } \\
\text { les critères de l'approche en } \\
\text { deux étapes (épreuve ELISA et } \\
\text { méthode de transfert Western } \\
\text { blot) } \\
\text { OU } \\
\text { - Érythème migrant observé } \\
\text { par un clinicien en l'absence } \\
\text { de résultats de laboratoire } \\
\text { indiquant une infection, mais } \\
\text { avec antécédents de résidence } \\
\text { ou de voyage dans une région } \\
\text { endémique }\end{array}$ \\
\hline
\end{tabular}

En 2015, huit provinces participaient au Système :

Colombie-Britannique, Alberta, Manitoba, Ontario,

Nouveau-Brunswick, Nouvelle-Écosse, île-du-Prince-Édouard et Terre-Neuve-et-Labrador. La Colombie-Britannique n'a pas fourni de données sur l'emplacement. Le Québec et la Saskatchewan n'ont fourni des données que par l'intermédiaire du SSMDOC.

\section{Analyse}

\section{Incidence au fil du temps}

L'incidence des cas signalés de la maladie de Lyme au Canada par année, province, groupe d'âge et sexe a été calculée par populations de 100 000. Les dénominateurs étaient des estimations de la population de recensement en date du

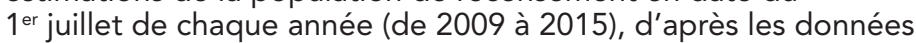
de Statistique Canada (9).

\section{Répartition saisonnière et géographique}

La répartition saisonnière des cas (par mois) a été effectuée à partir de la date d'apparition des signes ou des symptômes de la maladie de Lyme signalée dans le Système. L'emplacement géographique le plus probable pour l'éclosion des infections par la maladie de Lyme correspondait au centroïde de la subdivision de recensement (SDR) ou à la municipalité dans laquelle le patient était exposé à un risque de maladie de Lyme. Tous les cas d'antécédents de voyage (à l'intérieur ou à l'extérieur du Canada) dans les 30 jours suivant la déclaration n'ont pas été inclus dans les analyses géographiques. II a été impossible d'effectuer l'analyse géographique des infections contractées en Colombie-Britannique, car le lieu de l'acquisition n'a pas été signalé à l'Agence de la santé publique du Canada. 


\section{Caractéristiques démographiques et cliniques}

Les écarts entre les groupes d'âge, les sexes et les années de déclaration des manifestations cliniques ont été explorés par régression logistique au moyen de la version 24 du SPSS Statistics d'IBM (IBM, Chicago, État de l'Illinois, aux États-Unis). Dans des modèles distincts, les résultats étaient caractérisés par la présence ou l'absence des manifestations suivantes:

- érythème migrant (manifestation précoce);

- symptômes neurologiques et cardiaques, et érythèmes migrants multiples (stade disséminé précoce);

- arthrite (stade disséminé tardif).

Pour chaque modèle, les variables explicatives étaient le groupe d'âge, le sexe et l'année. Dans le cas de la variable " groupe d'âge ", deux intervalles d'âge, soit 10 et 15 ans, ont été examinés dans chaque modèle, et le groupe de référence était 0 à 9 ans et 0 à 14 ans, respectivement. La variable "province " a été incluse dans l'analyse pour tenir compte de la variabilité possible des déclarations entre les provinces. Le niveau de signification retenu pour les variables explicatives dans le modèle multivariable était de moins de 0,1 . Les modèles multivariables les plus restreints étaient recherchés par l'élimination de variables non significatives, jusqu'à ce que tous les facteurs du modèle soient significatifs $(p<0,05)$.

\section{Résultats}

\section{Incidence au fil du temps}

De 2009 à 2015, un total de 3012 cas de la maladie de Lyme ont été déclarés au Canada. Le nombre de cas signalés au Canada a plus que sextuplé, passant de 144 en 2009 à 917 en 2015. L'incidence nationale pour 100000 personnes a également augmenté, passant de 0,4 à 2,6 (tableau 2).

Tableau 2 : Nombre de cas de la maladie de Lyme signalés pour 100000 personnes par province et par année au Canada, de 2009 à 2015

\begin{tabular}{|c|c|c|c|c|c|c|c|}
\hline Province & 2009 & 2010 & 2011 & 2012 & 2013 & 2014 & 2015 \\
\hline \multicolumn{8}{|c|}{ Cas de la maladie de Lyme signalés au Canada $(n=3012)$} \\
\hline $\begin{array}{l}\text { Colombie- } \\
\text { Britannique }\end{array}$ & 0,2 & 0,2 & 0,4 & 0,4 & 0,1 & 0,1 & 0,4 \\
\hline Alberta* & 0,0 & 0,0 & 0,2 & 0,2 & 0,5 & 0,2 & 0,3 \\
\hline Saskatchewan* & 0,0 & 0,0 & 0,1 & 0,0 & 0,1 & 0,0 & 0,0 \\
\hline Manitoba & 0,4 & 1,0 & 1,0 & 1,5 & 2,3 & 2,7 & 2,4 \\
\hline Ontario & 0,8 & 0,7 & 1,0 & 1,4 & 2,4 & 1,7 & 3,1 \\
\hline Québec & 0,2 & 0,1 & 0,4 & 0,5 & 1,7 & 1,5 & 1,9 \\
\hline $\begin{array}{l}\text { Nouveau- } \\
\text { Brunswick }\end{array}$ & 0,0 & 0,3 & 0,7 & 0,9 & 0,7 & 0,7 & 1,7 \\
\hline Nouvelle-Écosse & 1,7 & 1,8 & 5,7 & 5,4 & 16,2 & 12,1 & 26,1 \\
\hline $\begin{array}{l}\text { Île-du-Prince- } \\
\text { Édouard }\end{array}$ & 0,0 & 0,0 & 0,7 & 1,4 & 0,0 & 0,0 & 2,7 \\
\hline $\begin{array}{l}\text { Terre-Neuve-et- } \\
\text { Labrador* }\end{array}$ & 0,0 & 0,2 & 0,0 & 0,0 & 0,0 & 0,0 & 0,4 \\
\hline Canada & 0,4 & 0,4 & 0,8 & 1,0 & 1,9 & 1,5 & 2,6 \\
\hline \multicolumn{8}{|c|}{ Cas de la maladie de Lyme acquis au Canada ${ }^{\dagger}(n=2004)$} \\
\hline Manitoba & 0,3 & 0,6 & 0,6 & 1,0 & 2,0 & 2,4 & 2,3 \\
\hline Ontario & 0,5 & 0,5 & 0,8 & 0,8 & 2,1 & 1,3 & 2,7 \\
\hline
\end{tabular}

Tableau 2 : Nombre de cas de la maladie de Lyme signalés pour 100000 personnes par province et par année au Canada, de 2009 à 2015 (suite)

\begin{tabular}{|c|c|c|c|c|c|c|c|}
\hline Province & 2009 & 2010 & 2011 & 2012 & 2013 & 2014 & 2015 \\
\hline \multicolumn{8}{|c|}{ Cas de la maladie de Lyme signalés au Canada ( $n=3012$ ) (suite) } \\
\hline $\begin{array}{l}\text { Nouv } \\
\text { Bruns }\end{array}$ & 0,0 & 0,3 & 0,4 & 0,7 & 0,7 & 0,5 & 1,5 \\
\hline Nouvelle-Écosse & 1,5 & 1,5 & 5,2 & 5,3 & 16,1 & 12,1 & 26,1 \\
\hline $\begin{array}{l}\text { Île-du-Prince- } \\
\text { Édouard }\end{array}$ & 0,0 & 0,0 & 0,0 & 0,7 & 0,0 & 0,0 & 0,0 \\
\hline \multicolumn{8}{|c|}{$\begin{array}{l}\text { Tous les cas signalés par l'Alberta, la Saskatchewan et Terre-Neuve-et-Labrador étaient liés à des } \\
\text { voyages } \\
\text { +L'information sur l'acquisition de la maladie de Lyme à l'intérieur du Canada a été fournie par } \\
\text { certaines des provinces participant au Système (Alberta, Manitoba, Ontario, Nouveau-Brunswick, } \\
\text { Nouvelle-Écosse et Île-du-Prince-Édouard). Cependant, seuls les cas de la maladie de Lyme } \\
\text { acquis dans la province d'origine ont été inclus }\end{array}$} \\
\hline
\end{tabular}

Parmi les cas acquis au Canada, 63,9\% étaient des cas confirmés et $36,1 \%$ étaient des cas probables (tableau 3 ).

Tableau 3 : Classification (confirmés et probables) de tous les cas signalés de la maladie de Lyme et des cas acquis au Canada*, de 2009 à 2015

\begin{tabular}{|c|c|c|c|c|c|c|c|c|}
\hline \multirow{2}{*}{$\begin{array}{c}\text { Classification } \\
\text { Year }\end{array}$} & \multicolumn{8}{|c|}{ Nombre (pourcentage de cas) } \\
\hline & 2009 & 2010 & 2011 & 2012 & 2013 & 2014 & 2015 & Total \\
\hline \multicolumn{9}{|c|}{ Cas de la maladie de Lyme signalés au Canada ( $n=3012)$} \\
\hline $\begin{array}{l}\text { Cas } \\
\text { confirmés }\end{array}$ & $\begin{array}{r}115 \\
(79,9 \%) \\
\end{array}$ & $\begin{array}{r}109 \\
(76,2 \%) \\
\end{array}$ & $\begin{array}{r}195 \\
(73,3 \%) \\
\end{array}$ & $\begin{array}{r}232 \\
(68,6 \%) \\
\end{array}$ & $\begin{array}{r}485 \\
(71,1 \%) \\
\end{array}$ & $\begin{array}{r}334 \\
(64,0 \%) \\
\end{array}$ & $\begin{array}{r}651 \\
(71,0 \%) \\
\end{array}$ & $\begin{array}{r}2121 \\
(70,4 \%) \\
\end{array}$ \\
\hline $\begin{array}{l}\text { Cas } \\
\text { probables }\end{array}$ & $\begin{array}{r}29 \\
20,1 \%) \\
\end{array}$ & $\begin{array}{r}34 \\
(23,8 \%) \\
\end{array}$ & $\begin{array}{r}71 \\
(26,7 \%) \\
\end{array}$ & $\begin{array}{r}106 \\
(31,4 \%) \\
\end{array}$ & $\begin{array}{r}197 \\
(28,9 \%) \\
\end{array}$ & $\begin{array}{r}188 \\
(36,0 \%) \\
\end{array}$ & $\begin{array}{r}266 \\
(29,0 \%) \\
\end{array}$ & $\begin{array}{r}891 \\
(29,6 \%) \\
\end{array}$ \\
\hline Total & 144 & 143 & 266 & 338 & 682 & 522 & 917 & 3012 \\
\hline \multicolumn{9}{|c|}{ Cas de la maladie de Lyme acquis au Canada $(n=2015)$} \\
\hline $\begin{array}{l}\text { Cas } \\
\text { confirmés }\end{array}$ & $\begin{array}{r}56 \\
(70,9 \%) \\
\end{array}$ & $\begin{array}{r}56 \\
(65,1 \%) \\
\end{array}$ & $\begin{array}{r}96 \\
(60,0 \%) \\
\end{array}$ & $\begin{array}{r}129 \\
(58,1 \%) \\
\end{array}$ & $\begin{array}{r}286 \\
(61,1 \%) \\
\end{array}$ & $\begin{array}{r}198 \\
(59,5 \%) \\
\end{array}$ & $\begin{array}{r}467 \\
(70,0 \%) \\
\end{array}$ & $\begin{array}{r}1288 \\
(63,9 \%) \\
\end{array}$ \\
\hline $\begin{array}{l}\text { Cas } \\
\text { probables }\end{array}$ & $\begin{array}{r}23 \\
(29,1 \%)\end{array}$ & $\begin{array}{r}30 \\
(34,9 \%)\end{array}$ & $\begin{array}{r}64 \\
(40,0 \%)\end{array}$ & $\begin{array}{r}93 \\
(41,9 \%)\end{array}$ & $\begin{array}{r}182 \\
(38,9 \%)\end{array}$ & $\begin{array}{r}135 \\
(40,5 \%)\end{array}$ & $\begin{array}{r}200 \\
(30,0 \%)\end{array}$ & $\begin{array}{r}727 \\
(36,1 \%) \\
\end{array}$ \\
\hline Total & 79 & 86 & 160 & 222 & 468 & 333 & 667 & 2015 \\
\hline
\end{tabular}

+ L'information sur les cas de la maladie de Lyme acquis au Canada (nombre et pourcentage) a été fournie par les provinces participant au Système (Alberta, Manitoba, Ontario, Nouveau-Brunswick, Nouvelle-Écosse et île-du-Prince-Édouard). Pour être précis, tous les cas déclarés comme ayant été acquis au Canada, à l'intérieur ou à l'extérieur de la province, ont été inclus

La hausse de l'incidence de la maladie de Lyme au Canada est principalement due à une hausse du nombre d'infections localement contractées, 79 en 2009 et 667 en 2015.

Au cours de l'étude, l'information sur le pays d'exposition était accessible pour 1950 cas. De ces cas, 1709 (87,6\%) ont été acquis au Canada. La majorité des cas pour lesquels l'information sur le lieu de l'acquisition était disponible ont été signalés comme ayant été acquis dans des régions reconnues à risque. Cependant, certains cas ont été déclarés comme ayant été acquis au Canada, mais à l'extérieur des zones à risque connues. Le reste de ces cas, 241 (12,4\%), ont été acquis à l'étranger; plus de la moitié de ces cas liés à des voyages ont été signalés comme ayant été acquis aux États-Unis.

\section{Répartition saisonnière}

En ce qui concerne les provinces participant au Système, le mois d'apparition de la maladie de Lyme acquise au Canada était connu pour 2010 cas. De ce nombre, $96 \%$ ont été déclarés entre mai et novembre, avec un pic constant du nombre de cas 
observé en juillet. La plupart des cas ont été signalés durant les mois estivaux de juin $(20,7 \%)$, de juillet $(35,4 \%)$ et d'août (17,3\%) (figure 1). En 2015, un nombre accru de cas ont été signalés comme étant apparus en novembre et en décembre, ce qui laisse entendre que les tiques ont été actives plus tard dans la saison cette année-là, comparativement aux autres années.

Figure 1 : Mois d'apparition de la maladie de Lyme dans les cas d'infection localement acquis : Canada, de 2009 à $2015(n=2$ 010)

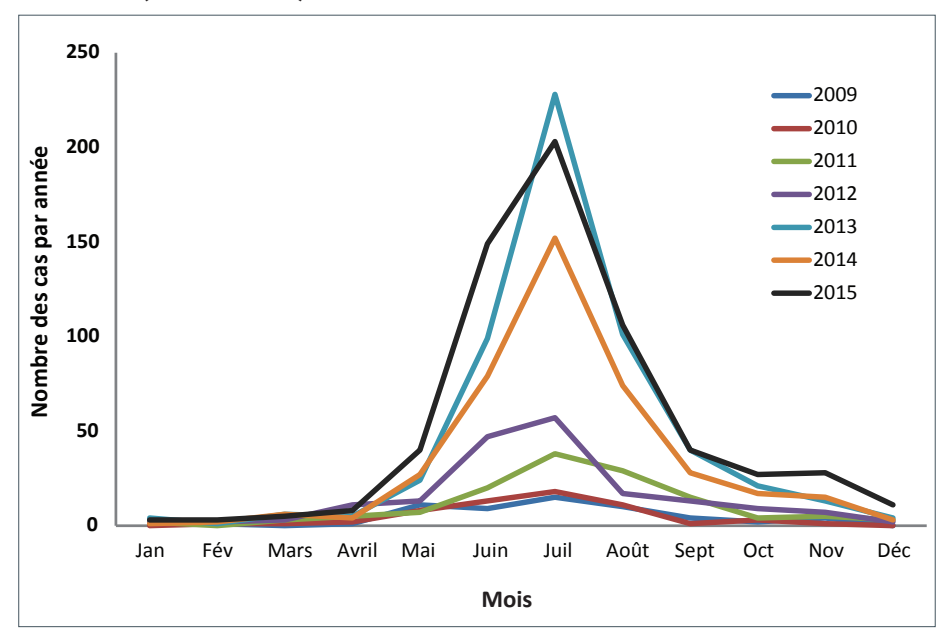

\section{Répartition géographique}

Entre 2009 et 2015, le Manitoba et les provinces situées plus à l'Est ont enregistré une hausse de l'incidence de la maladie de Lyme, la hausse la plus importante ayant eu lieu en Nouvelle-Écosse. Dans les provinces de l'Ouest, l'incidence est restée faible et stable. Tous les cas signalés par l'Alberta, la Saskatchewan et Terre-Neuve-et-Labrador ont été contractés à l'extérieur des provinces en question, au Canada ou à l'étranger. L'année 2014 a connu une baisse de l'incidence dans la plupart des provinces, suivie par une hausse en 2015.

En ce qui concerne les provinces participant au Système, le nombre de municipalités où des cas de la maladie de Lyme ont été acquis au Canada a plus que quintuplé au cours de l'étude, passant de 21 en 2009 à 109 en 2015 (tableau 4).

Les lieux déclarés d'acquisition de la maladie de Lyme au Canada sont présentés dans la figure 2. Le nombre de régions à risque pour la maladie de Lyme a augmenté au fil du temps au Manitoba, en Ontario, au Québec, au Nouveau-Brunswick et en Nouvelle-Écosse (1).

\section{Caractéristiques démographiques et cliniques}

Au cours de l'étude, le nombre de cas signalés de la maladie de Lyme a été légèrement plus élevé chez les hommes ( $n=1688$, $56 \%$ des cas) que chez les femmes ( $n=1316,44 \%$ des cas). L'âge moyen de tous les cas déclarés était de 45 ans (IC à $95 \%$, 44,3 à 45,8). L'incidence pour 100000 personnes a révélé un

Tableau 4 : Nombre de cas de la maladie de Lyme et de municipalités d'acquisition, déclarés chaque année par les provinces participant au Système de surveillance accrue de la maladie de Lyme, au Canada de 2009 à 2015

\begin{tabular}{|c|c|c|c|c|c|c|c|c|}
\hline \multicolumn{2}{|c|}{ Province } & \multirow{2}{*}{$\begin{array}{r}2009 \\
\text { n.d. }\end{array}$} & \multirow{2}{*}{$\begin{array}{r}2010 \\
\text { n.d. }\end{array}$} & \multirow{2}{*}{\begin{tabular}{r|}
2011 \\
n.d.
\end{tabular}} & \multirow{2}{*}{$\begin{array}{r}2012 \\
\text { n.d. }\end{array}$} & \multirow{2}{*}{$\begin{array}{r}2013 \\
\text { n.d. }\end{array}$} & \multirow{2}{*}{$\begin{array}{r}2014 \\
\text { n.d. }\end{array}$} & \multirow{2}{*}{$\begin{array}{r}2015 \\
\text { n.d. }\end{array}$} \\
\hline & Cas (nombre) & & & & & & & \\
\hline Colombie-Britannique & Municipalité (nombre) & n.d. & n.d. & n.d. & n.d. & n.d. & n.d. & n.d. \\
\hline \multirow{2}{*}{ Alberta } & Cas (nombre) & 0 & 0 & 0 & 0 & 0 & 0 & 0 \\
\hline & Municipalité (nombre) & 0 & 0 & 0 & 0 & 0 & 0 & 0 \\
\hline \multirow{2}{*}{ Manitoba } & Cas (nombre) & 0 & 4 & 7 & 12 & 19 & 28 & 22 \\
\hline & Municipalité (nombre) & 0 & 2 & 4 & 3 & 9 & 17 & 12 \\
\hline \multirow{2}{*}{ Ontario } & Cas (nombre) & 37 & 38 & 85 & 92 & 184 & 144 & 323 \\
\hline & Municipalité (nombre) & 18 & 18 & 31 & 37 & 54 & 45 & 74 \\
\hline \multirow{2}{*}{ Nouveau-Brunswick* } & Cas (nombre) & n.d. & 0 & 3 & 5 & 1 & 3 & 7 \\
\hline & Municipalité (nombre) & n.d. & 0 & 1 & 1 & 1 & 2 & 2 \\
\hline \multirow{2}{*}{ Nouvelle-Écosse } & Cas (nombre) & 13 & 17 & 50 & 50 & 151 & 114 & 239 \\
\hline & Municipalité (nombre) & 3 & 5 & 10 & 7 & 13 & 15 & 21 \\
\hline \multirow{2}{*}{ Île-du-Prince-Édouard* } & Cas (nombre) & n.d. & n.d. & n.d. & 1 & 0 & 0 & 0 \\
\hline & Municipalité (nombre) & n.d. & n.d. & n.d. & 1 & 0 & 0 & 0 \\
\hline \multirow{2}{*}{ Terre-Neuve-et-Labrador } & Cas (nombre) & 0 & 0 & 0 & 0 & 0 & 0 & 0 \\
\hline & Municipalité (nombre) & 0 & 0 & 0 & 0 & 0 & 0 & 0 \\
\hline \multirow{2}{*}{ Total } & Cas (nombre) & 50 & 59 & 145 & 160 & 355 & 289 & 591 \\
\hline & Municipalité (nombre) & 21 & 25 & 46 & 49 & 77 & 79 & 109 \\
\hline
\end{tabular}

Abréviation : n.d., non disponible

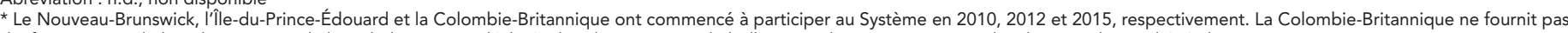
d'information sur le lieu d'acquisition de la maladie. Les cas déclarés dans les provinces de l'Alberta et de Terre-Neuve-et-Labrador sont des cas liés à des voyages uniquement 
Figure 2 : Lieux déclarés d'acquisition de la maladie de Lyme au Canada, de 2009 à 2015

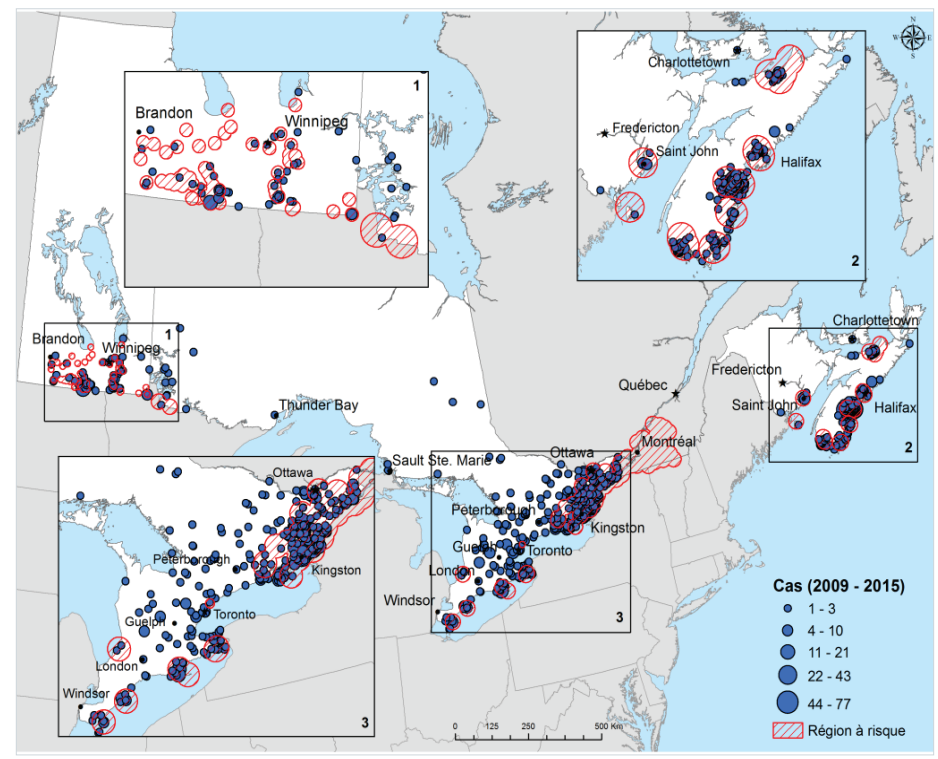

Légende : La taille de chaque cercle représente le nombre de cas acquis dans une municipalité donnée. Le centre de chaque cercle est le centroïde de la municipalité probable d'acquisition. Les données sur le lieu d'acquisition ne sont pas disponibles pour les cas déclarés en ColombieBritannique, en Saskatchewan et au Québec. En outre, les cas déclarés dans les provinces de l'Alberta et de Terre-Neuve-et-Labrador sont des cas liés à des voyages uniquement. Les zones hachurées indiquent les régions à risque pour la maladie de Lyme. Dans ces régions, la surveillance sur le terrain semble indiquer que les populations du vecteur de la maladie de Lyme Ixodes scapularis ont commencé à s'établir (1)

modèle bimodal avec une incidence plus élevée chez les adultes de 45 à 74 ans et chez les enfants de 5 à 9 ans. Dans tous les groupes d'âge, l'incidence était plus élevée chez les hommes que chez les femmes, à l'exception du groupe d'âge de 10 à 14 ans (figure 3 ).

Figure 3 : Incidence de la maladie de Lyme par groupe d'âge et par sexe au Canada, de 2009 à $2015(n=3004)$

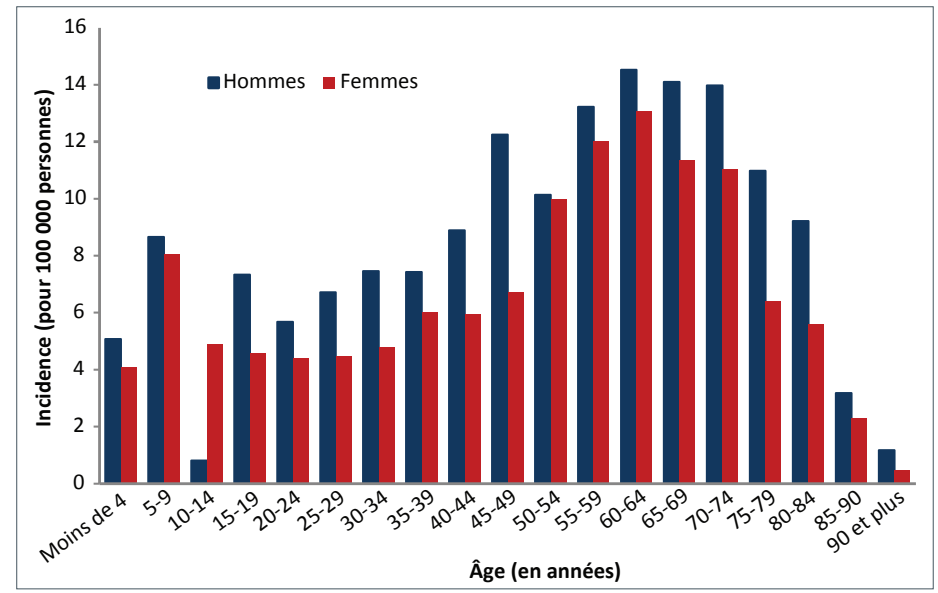

Des renseignements cliniques étaient accessibles pour 1657 (55\%) des cas déclarés de la maladie de Lyme acquise au Canada. L'érythème migrant (74,2 \%) et l'arthrite (35,7 \%) constituaient les manifestations les plus courantes, et les lésions d'érythème migrant multiples $(5,9 \%)$ et les symptômes cardiaques (3,6\%), les moins courantes (figure 4). De multiples manifestations cliniques ont été signalées dans 33,2\% des cas.
Figure 4 : Pourcentage de manifestations cliniques de la maladie de Lyme acquise au Canada, de 2009 à 2015 $(n=1657)$

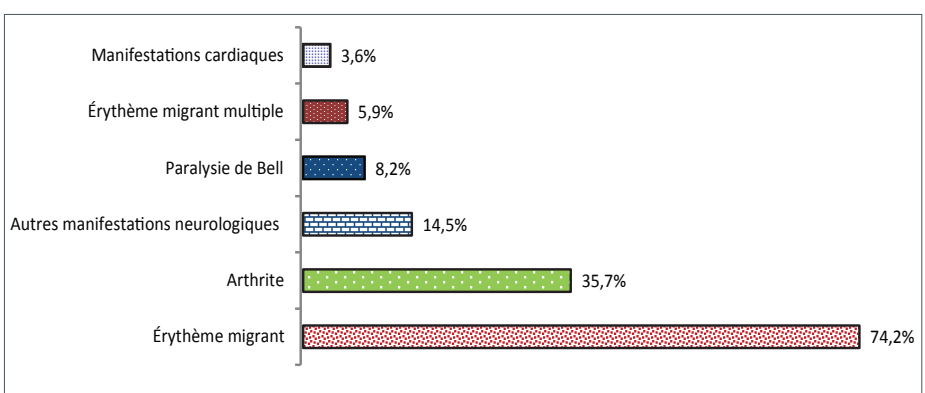

Les proportions relatives des diverses manifestations cliniques ont été quelque peu différentes pour les cas signalés chez les enfants de moins de 15 ans comparativement aux autres groupes d'âge. Dans ce groupe d'âge, les lésions d'érythème migrant étaient plus couramment signalées, tandis que les manifestations neurologiques et cardiaques étaient moins fréquentes que dans les groupes plus âgés (figure 5). Les manifestations cardiaques étaient plus fréquemment signalées chez les adultes de 20 à 44 ans que dans les autres groupes d'âge.

Figure 5 : Pourcentage de cas déclarés de la maladie de Lymel par manifestation clinique et groupe d'âge au Canada, de 2009 à $2015(n=1657)$

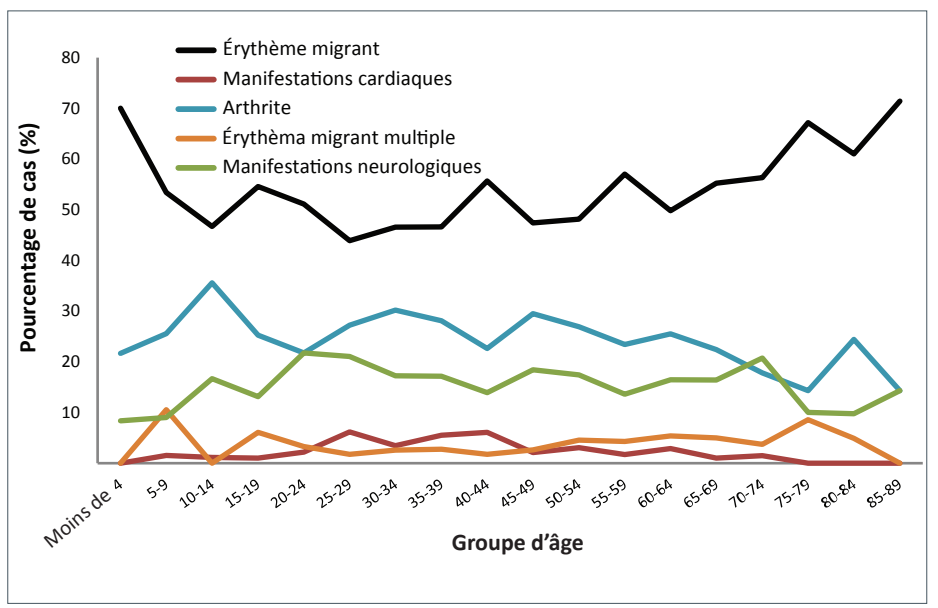

Abréviation : \%, pourcentage

Cas déclarés dans le Système de surveillance accrue de la maladie de Lyme

Dans l'analyse multivariée des manifestations cliniques, les cas de maladie de Lyme au stade précoce (érythème migrant uniquement) étaient plus fréquemment déclarés chez les enfants de 0 à 9 ans que chez les patients de 10 à 19 ans et de 30 à 39 ans $(p<0,05)$ (tableau 5$)$. En ce qui concerne les manifestations disséminées précoces, les jeunes adultes de 20 à 29 ans ont déclaré plus de manifestations neurologiques ou cardiaques ou d'érythèmes migrants multiples que le groupe $d$ 'âge de référence de 0 à 9 ans $(p<0,05)$. En ce qui concerne les manifestations disséminées tardives, l'arthrite a été plus fréquemment déclarée chez les enfants de moins de 15 ans que dans les autres groupes d'âge.

Les cas déclarés de la maladie de Lyme au stade disséminé tardif étaient significativement plus élevés de 2009 à 2011 qu'en 2015 $(p<0,01)$. Ces données corroborent l'observation selon 
Tableau 5 : Modèles finaux de régression logistique multivariée prévoyant l'apparition des manifestations cliniques de la maladie de Lyme chez les patients au Canada, de 2009 à 2015 ( $n=1657)$

\begin{tabular}{|c|c|c|c|c|c|}
\hline $\begin{array}{l}\text { Variables } \\
\text { explicatives }\end{array}$ & Estimation & $\begin{array}{l}\text { Rapport } \\
\text { des cotes }\end{array}$ & $\begin{array}{l}\text { IC à } \\
95 \%\end{array}$ & $\begin{array}{l}\text { Test } \\
\text { de } \\
\text { Wald }\end{array}$ & Valeur $\mathrm{P}$ \\
\hline \multicolumn{6}{|c|}{ Maladie de Lyme au stade précoce (érythème migrant) } \\
\hline $\begin{array}{l}10 \text { à } 19 \text { ans } \\
\text { contre } 0 \text { à } \\
9 \text { ans }\end{array}$ & $-0,490$ & 0,613 & $\begin{array}{r}0,378 \text { à } \\
0,994\end{array}$ & 3,932 & 0,047 \\
\hline $\begin{array}{l}30 \text { à } 39 \text { ans } \\
\text { contre } 0 \text { à } \\
9 \text { ans }\end{array}$ & $-0,513$ & 0,599 & $\begin{array}{r}0,378 \text { à } \\
0,950\end{array}$ & 4,753 & 0,029 \\
\hline \multicolumn{6}{|c|}{$\begin{array}{l}\text { Stade disséminé précoce (symptômes neurologiques et cardiaques, } \\
\text { érythème migrant multiple) }\end{array}$} \\
\hline $\begin{array}{l}\text { Homme/ } \\
\text { femme }\end{array}$ & $-0,272$ & 0,762 & $\begin{array}{r}0,585 \text { à } \\
0,992\end{array}$ & 4,083 & 0,043 \\
\hline $\begin{array}{l}20 \text { à } 29 \text { ans } \\
\text { contre } 0 \text { à } \\
9 \text { ans }\end{array}$ & 0,678 & 1,969 & $\begin{array}{r}1,071 \text { à } \\
3,623\end{array}$ & 4,751 & 0,029 \\
\hline $\begin{array}{l}2010 \text { contre } \\
2015\end{array}$ & $-0,555$ & 0,574 & $\begin{array}{r}0,392 \text { à } \\
0,841\end{array}$ & 8,137 & 0,004 \\
\hline \multicolumn{6}{|c|}{ Stade disséminé tardif (arthrite) } \\
\hline $\begin{array}{l}\text { Homme/ } \\
\text { femme }\end{array}$ & 0,246 & 1,279 & $\begin{array}{r}1,031 \text { à } \\
1,586\end{array}$ & 5,012 & 0,025 \\
\hline $\begin{array}{l}75 \text { ans et } \\
\text { plus contre } \\
0 \text { à } 14 \text { ans }\end{array}$ & $-0,691$ & 0,501 & $\begin{array}{r}0,283 \text { à } \\
0,888\end{array}$ & 5,604 & 0,018 \\
\hline $\begin{array}{l}\text { Année } 2011 \\
\text { contre } 2015\end{array}$ & 0,565 & 1,759 & $\begin{array}{r}1,221 \text { à } \\
2,533\end{array}$ & 9,200 & 0,002 \\
\hline $\begin{array}{l}\text { Année } 2010 \\
\text { contre } 2015\end{array}$ & 0,774 & 2,168 & $\begin{array}{r}1,630 \text { à } \\
2,883\end{array}$ & 28,777 & 0,000 \\
\hline $\begin{array}{l}\text { Année } 2009 \\
\text { contre } 2015\end{array}$ & 0,472 & 1,603 & $\begin{array}{r}1,168 \text { à } \\
2,199\end{array}$ & 8,554 & 0,003 \\
\hline
\end{tabular}

Abréviation : IC, intervalle de confiance

laquelle un plus grand nombre de cas de la maladie de Lyme au stade disséminé précoce ont été déclarés en 2015 qu'en 2010 $(p<0,01)$.

\section{Discussion}

Depuis que la maladie de Lyme est devenue une maladie à déclaration obligatoire à l'échelle nationale en 2009, le nombre de cas signalés de la maladie a continué d'augmenter, passant de 0,4 à 2,6 pour 100000 personnes. Au cours de cette période de sept ans, la plupart des cas ont été déclarés dans trois provinces, soit I'Ontario, le Québec et la Nouvelle-Écosse, et la plupart ont été acquis localement. La hausse de la répartition géographique des cas de maladie de Lyme cadre avec l'expansion continue de l'aire de répartition des tiques à pattes noires, laquelle est probablement liée en partie aux effets du réchauffement climatique sur l'étendue de la propagation des tiques vectrices I. scapularis dans l'est et le centre du Canada (10). Cependant, I'incidence reste faible et stable dans les provinces de l'Ouest, soit la Colombie-Britannique, I'Alberta et la Saskatchewan. Cela s'explique par le fait qu'en Colombie-Britannique, un plus petit nombre de tiques à pattes noires, vecteur de la maladie de Lyme dans cette région, sont infectées par la bactérie responsable de la maladie des tiques à pattes noires que l'on trouve dans l'est et le centre du Canada. En Alberta et en Saskatchewan, malgré des efforts accrus, aucune population de tiques à pattes noires n'a été repérée.

Les dates d'apparition déclarées de la maladie étaient plus nombreuses durant les mois d'été, ce qui correspond à la période d'activité des tiques I. scapularis à la recherche d'hôtes (11) et chevauche la période à laquelle la plupart des Canadiens s'adonnent à des activités de plein air.

Chez les adultes, l'incidence était la plus élevée chez ceux âgés de 55 à 74 ans. Chez les enfants, I'incidence était la plus élevée chez ceux âgés de 5 à 9 ans. Ces données correspondent aux tendances démographiques observées aux États-Unis $(3,6)$ et peuvent être utiles pour cibler les messages de sensibilisation $(12,13)$. Un plus petit nombre de cas de la maladie de Lyme au stade disséminé tardif a été signalé en 2015, comparativement aux années 2009 à 2011, ce qui semble indiquer une sensibilisation accrue ainsi qu'un diagnostic et une déclaration des cas de maladie de Lyme plus précoces au fil du temps.

\section{Forces et faiblesses}

Cette étude résume les données du Système de surveillance des maladies à déclaration obligatoire du Canada, complétées dans la plupart des provinces par des renseignements provenant du Système de surveillance accrue de la maladie de Lyme. À l'aide de ces données, nous sommes en mesure de suivre les tendances spatiales et temporelles associées à l'évolution de I'incidence de la maladie de Lyme au Canada, et d'étudier la propagation géographique des risques de maladie de Lyme ainsi que l'efficacité des mesures de santé publique.

L'interprétation de ces résultats se heurte à trois limites principales. Tout d'abord, il est probable que les incidences au fil du temps constituent des estimations prudentes, car certains cas de maladie de Lyme peuvent ne pas être diagnostiqués, et certains cas probables peuvent être sous-déclarés. Ensuite, les renseignements sur le lieu d'acquisition de l'infection par la maladie de Lyme, localement ou pendant des voyages à l'extérieur du pays, sont des estimations, car les provinces n'ont pas toutes fourni ces données. Enfin, en raison des ressources limitées, la surveillance des tiques sur le terrain servant à repérer l'expansion des régions à risque n'est sans doute pas à jour dans de nombreux endroits, ce qui pourrait avoir une incidence sur la classification des cas.

\section{Conclusion}

Le nombre de cas déclarés de la maladie de Lyme a continué d'augmenter au Canada au cours des dernières années, tout comme la portée géographique des tiques qui transportent la bactérie responsable de la maladie. La surveillance continue, les stratégies de prévention ainsi que la détection et le traitement précoces de la maladie permettront réduire au minimum les répercussions de la maladie de Lyme au Canada.

\section{Déclaration d'auteurs}

S.G., J.K.K. - Collecte et conservation des données, conceptualisation, méthodologie, analyse formelle, rédaction ébauche originale, rédaction - examen et révision 
L.R.L., N.O. - Conceptualisation, méthodologie, rédaction examen et révision

S.F., J.B., S.H., C.G., M.A.L., C.R., L.H., S.G-D., L.L., A.N.S., E.G. : rédaction - examen et révision

\section{Conflit d'intérêt}

Aucun.

\section{Remerciements}

Les auteurs remercient tous les travailleurs de la santé publique à l'échelle provinciale et régionale qui recueillent des données et les déclarent au SSMDOC, ainsi que Yann Pelcat de l'Agence de la santé publique du Canada pour la préparation de la figure 5.

\section{Financement}

Cette étude a été appuyée par l'Agence de la santé publique du Canada et tous les organismes de santé publique provinciaux qui ont contribué à la collecte de données.

\section{Références}

1. Ogden NH, Koffi KJ, Pelcat Y, Lindsay LR. Risque environnemental pour la maladie de Lyme dans l'est et le centre du Canada : un sommaire d'informations récentes en matière de surveillance. Relevé des maladies transmissibles au Canada. 2014;40(5):77-86. http://www. phac-aspc. gc.ca/publicat/ccdr-rmtc/14vol40/dr-rm40-05/assets/ pdf/14vol40_05-fra.pdf

2. Hatchette TF, Davis I, Johnston BL. La maladie de Lyme : Diagnostic clinique et traitement. Relevé des maladies transmissibles au Canada. Rep. 2014;40:215-31. https:// www.canada.ca/fr/sante-publique/services/rapportspublications/releve-maladies-transmissibles-canada-rmtc/ numero-mensuel/2014-40/rmtc-volume-40-11-29-mai-2014/ rmtc-volume-40-11-29-mai-2014.html

3. Bacon RM, Kugeler KJ, Mead P; Centers for Disease Control and Prevention (CDC). Surveillance for Lyme disease-United States, 1992-2006. MMWR Surveill Summ. 2008;57(10):1-9. PubMed (https://www.ncbi.nlm. nih.gov/entrez/query.fcgi?cmd=Retrieve\&db=PubMed\&li st_uids=18830214\&dopt=Abstract).

4. Centers for Disease Control and Prevention. Three sudden cardiac deaths associated with Lyme carditis - United States, November 2012-July 2013. MMWR Morb. Mortal. Wkly. Rep. 2013;62(49):993-6. PubMed (https://www.ncbi.nlm. nih.gov/entrez/query.fcgi?cmd=Retrieve \&db=PubMed\&li st_uids=24336130\&dopt=Abstract).

5. Agence de la santé publique du canada. Surveillance de la maladie de Lyme au Canada. Ottawa: Agence de la santé publique du canada; 2017. https://www.canada.ca/fr/santepublique/services/maladies/maladie-lyme/surveillancemaladie-lyme.html\#a2 [Consulté le 13 avril 2017].

6. Ogden NH, Koffi KJ, Lindsay LR, Fleming S, Monbourquette DC, Sanford C, Badcock J, Gad RR, Jain-Sheehan N, Moore S, Russell C, Hobbs L, Baydack R, Graham-Derham S, Lachance L, Simmonds K, Scott AN. Surveillance de la maladie de Lym au Canada, 2009 à 2012. Relevé des maladies transmissibles au Canada. 2015;41(6):152-68. https://www.canada.ca/fr/sante-publique/services/rapportspublications/releve-maladies-transmissibles-canada-rmtc/ numero-mensuel/2015-41/rmtc-volume-41-06-4-juin-2015/ rmtc-volume-41-06-4-juin-2015-2.html

7. Agence de la santé publique du Canada. La maladie de Lyme au Canada - cadre fédéral. Ottawa: Agence de la santé publique du Canada; 2017. https://www.canada.ca/fr/ sante-publique/services/publications/maladies-et-affections/ maladie-lyme-canada-cadre-federal.html

8. Agence de la santé publique du Canada. Définitions nosologiques des maladies transmissibles faisant l'objet d'une surveillance nationale - 2009. Ottawa: Agence de la santé publique du Canada; 2017. https://www.canada. $\mathrm{ca} / \mathrm{fr} /$ sante-publique/services/rapports-publications/ releve-maladies-transmissibles-canada-rmtc/numeromensuel/2009-35/definitions-nosologiques-maladiestransmissibles-surveillance-nationale.html [Consulté le 17 août 2017].

9. Statistique Canada. CANSIM par sujet : Population et démographie. Ottawa: Statistique Canada; 2017. http:// www5.statcan.gc.ca/cansim/a33?lang=fra\&spMode=master\& themelD=3867\&RT=TABLE [Consulté le 13 avril 2017].

10. Leighton PA, Koffi KJ, Pelcat $Y$, Lindsay LR, Ogden NH. Predicting the speed of tick invasion: an empirical model of range expansion for the Lyme disease vector Ixodes scapularis in Canada. J Appl Ecol. 2012;49(2):457-64. DOI (http://dx.doi.org/10.1111/j.1365-2664.2012.02112.x).

11. Kurtenbach K, Hanincová K, Tsao JI, Margos G, Fish D, Ogden NH. Fundamental processes in the evolutionary ecology of Lyme borreliosis. Nat Rev Microbiol. 2006;4(9):660-9. DOI (http://dx.doi.org/10.1038/ nrmicro1475).

12. Liang W, Shediac-Rizkallah MC, Celentano DD, Rohde C. A population-based study of age and gender differences in patterns of health-related behaviors. Am J Prev Med 1999;17(1):8-17. DOI (http://dx.doi.org/10.1016/S07493797(99)00040-9). PubMed (https://www.ncbi.nlm.nih. gov/entrez/query.fcgi?cmd=Retrieve \&db=PubMed\&lis t_uids=10429747\&dopt=Abstract).

13. Agence de la santé publique du Canada. Prévention de la maladie de Lyme. Ottawa: Agence de la santé publique du Canada; 2017. https://www.canada.ca/fr/sante-publique/ services/maladies/maladie-lyme/prevention-maladie-lyme. html [Consulté le 17 août 2017]. 